\title{
Evaluation of the novel Tanita body-fat analyser to measure body composition by comparison with a four-compartment model
}

\author{
Susan A. Jebb*, Timothy J. Cole, Deanne Doman, Peter R. Murgatroyd and Andrew M. Prentice \\ MRC Human Nutrition Research (formerly Dunn Nutritional Laboratory), Downhams Lane, Cambridge CB4 IXJ, UK
}

(Received 18 January 1999 - Revised 30 July 1999 - Accepted 17 August 1999)

\begin{abstract}
The Tanita body-fat analyser is a novel device to estimate body fat, based on the principles of bioelectrical impedance. It differs from other impedance systems which use surface electrodes in that the subjects stand bare-footed on a metal sole-plate which incorporates the electrodes, hence impedance is measured through the legs and lower trunk. In 104 men and 101 women (16-78 years and BMI $16-41 \mathrm{~kg} / \mathrm{m}^{2}$ ) the mean bias in body-fat mass measured using the Tanita body-fat analyser was $0.8(2 \mathrm{SD} 7.9) \mathrm{kg}$ relative to a four-compartment model. This is comparable to the other prediction techniques tested (conventional tetrapolar impedance -1.3 (2SD 6.9) $\mathrm{kg}$, skinfold thicknesses 0.3 (2SD 7.4) kg, and BMI-based formulas -0.2 (2SD 9.0) kg and -0.6 (2SD $8.5) \mathrm{kg}$ ), but the agreement was poorer than for 'reference' methods to measure body fat (density $0 \cdot 2(2 \mathrm{SD} 3 \cdot 7) \mathrm{kg}$, total body water $-0.9(2 \mathrm{SD} 3.4) \mathrm{kg}$ and dual-energy X-ray absorptiometry 0.1 $(2 \mathrm{SD} 5 \cdot 0) \mathrm{kg}$ ). The present paper also describes the derivation of a new prediction equation for the calculation of body composition from the Tanita body-fat analyser. The equation incorporates sex, age, and a log-transformation of height, weight and the measured impedance to predict body fat measured by a four-compartment model. This approach is recommended in the derivation of other prediction equations in body composition analysis. Using this novel prediction equation the residual standard deviations were $4.8 \%$ for men and $3.3 \%$ for women. A similar analysis using data collected with a conventional tetrapolar system yielded residual standard deviations of $4.3 \%$ for men and $3.1 \%$ for women. This demonstrates that the practical simplicity of the novel Tanita method is not associated with a clinically significant decrement in performance relative to a traditional impedance device.
\end{abstract}

Body composition: Body fat: Four-compartment model: Tanita

There is growing interest in the measurement of body composition, in particular the assessment of fat mass. The adverse health consequences of excess body fat are well documented and measurements of body fat are used in medical research as a marker of future risk. Other groups are interested in the composition of the body in relation to physical fitness.

Methods to measure body fat can be considered either as 'reference' or 'prediction' techniques (for reviews see Jebb \& Elia, 1993; Jebb, 1998). Reference methods such as body density, total body water or dual-energy X-ray absorptiometry (DXA), measure some physical property of the body, whilst prediction methods e.g. skinfold thicknesses and bioelectrical impedance analysis, use regression analysis to estimate the outcome of one or more reference techniques, traditionally total body water measurements for bioelectrical impedance and body density for skinfold thicknesses. Methods for estimating body fat from measures of weight and height alone have also been described (e.g.
Black et al. 1983; Garrow \& Webster, 1985). These methods therefore incorporate both their own inherent errors and those of the method from which they were derived. However, they generally offer the user a simpler and more convenient means of estimating body fat.

Body composition analysis takes a compartmental view of the body. In the classical two-compartment models the body is divided into fat and fat-free components. The fat component has a relatively homogeneous composition, but the fat-free mass is very heterogeneous, consisting of water, mineral, fat, protein and additional minor constituents. Twocompartment models must therefore assume that these components exist in a known relationship to each other. For example, the whole-body density method assumes known densities of fat and fat-free tissue and the total body water method assumes a known hydration fraction of fat-free tissue. However in practice there is considerable inter-individual variability. There are even intra-individual differences in the same subject studied on two or more 
Table 1. Characteristics of the subjects in the present study

(Mean values with standard deviations and ranges)

\begin{tabular}{|c|c|c|c|c|c|c|}
\hline & \multicolumn{3}{|c|}{ Male $(n 104)$} & \multicolumn{3}{|c|}{ Female ( $n$ 101) } \\
\hline & Mean & SD & Range & Mean & SD & Range \\
\hline Age (years) & 43.8 & $16 \cdot 0$ & $16-78$ & $40 \cdot 4$ & $13 \cdot 6$ & $18-70$ \\
\hline Height $(\mathrm{m})$ & 1.77 & 0.07 & $1.58-1.93$ & 1.65 & 0.06 & $1.44-1.83$ \\
\hline Weight (kg) & $81 \cdot 0$ & $17 \cdot 1$ & $50 \cdot 4-128 \cdot 8$ & $70 \cdot 3$ & $14 \cdot 8$ & $44 \cdot 7-105 \cdot 2$ \\
\hline $\mathrm{BMI}\left(\mathrm{kg} / \mathrm{m}^{2}\right)$ & 25.9 & $5 \cdot 3$ & $17 \cdot 4-40.5$ & 25.9 & 5.5 & $16 \cdot 1-39 \cdot 6$ \\
\hline
\end{tabular}

occasions, particularly in relation to the proportions of water and mineral in the fat-free compartment. This variability contributes to the absolute error of these methods.

DXA can be used to provide a three-compartment model, incorporating bone mineral, fat and fat-free soft tissue (Jebb, 1997). Furthermore, by combining measurements of body weight, density, total body water and bone mineral it is possible to derive a theoretically superior four-compartment model of body composition (Fuller et al. 1992). This overcomes the assumptions regarding the hydration and mineral fractions of fat-free tissue and has become accepted as an in vivo 'gold standard' (Jebb \& Elia, 1995). This multicompartment analysis provides a more accurate measure of absolute body composition than simpler models and is a more appropriate reference method for the development of prediction equations.

The Tanita body-fat analyser is a novel system to estimate body fat based on the principle of bioelectrical impedance analysis (Nunez et al. 1997). In contrast with traditional impedance devices, which use tetrapolar surface electrodes and a hand-to-foot current pathway, the Tanita system measures the voltage drop from foot to foot when a small alternating current is applied through contact with two metal foot plates. Weight is recorded automatically and height entered manually, whereas in conventional impedance devices both weight and height must be measured and entered. Using a conventional impedance machine subjects must be supine with surface electrodes connected at specific anatomical sites. The Tanita machine resembles a set of bathroom scales on which the subject stands barefoot and the machine displays weight and the percentage body fat. The system is designed for home-based use and is the first device to be sold directly to the public as a method to measure body fat.

The present study provides a comprehensive evaluation of a range of body composition techniques, both reference and prediction methods, in relation to a four-compartment model. In addition it includes the derivation of a new prediction equation to estimate body composition relative to a four-compartment model, for the novel Tanita body-fat analyser.

\section{Methods \\ Subjects}

Subjects were recruited from the Dunn Nutrition Centre volunteer databases and by local advertisements. All subjects were generally healthy, although some subjects were obese. Approval was obtained from the volunteer's general practitioner before participation in the study. The study was approved by the MRC Dunn Nutrition Unit ethical committee. Of those subjects who attended for measurement only five were unable to complete the measurement protocol. In each of these cases the subject was unable to tolerate the underwater weighing procedure. Data from these subjects were excluded from this analysis.

After these exclusions the total sample included 104 men and 101 women. Subjects were selected to provide a range of age and adiposity values (Table 1). The distribution of subjects by age and BMI is shown in Table 2. Data from all subjects and for all measurements are included in the analysis.

\section{Body composition measurements}

Measurements of body composition using each different technique were conducted according to standard procedures which have been extensively described elsewhere (Jebb \& Elia, 1993; Jebb et al. 1993) and are summarized here. All measurements, without exception, were performed by a single investigator (D. D.). Subjects had not eaten for at least $2 \mathrm{~h}$ before the measurement and each emptied their bladder immediately before the start of the measurements. In brief the procedures were as follows.

Weight and height. Weight was measured to the nearest $10 \mathrm{~g}$ using a digital scale. Subjects were weighed in their swimwear which had been previously weighed in order to correct the clothed body weight to nude. Height was

Table 2. Distribution of male and female subjects by age and BMI

\begin{tabular}{lrrrrr}
\hline & \multicolumn{5}{c}{ BMI $\left(\mathrm{kg} / \mathrm{m}^{2}\right)$} \\
\cline { 2 - 6 } Age (years) & $\leqslant 20$ & $20-24 \cdot 9$ & $25-29 \cdot 9$ & $\geqslant 30$ & Total \\
\hline Males & 2 & 6 & 2 & 1 & 11 \\
$\leqslant 24$ & 4 & 8 & 4 & 3 & 19 \\
$25-34$ & 2 & 7 & 11 & 5 & 25 \\
$35-44$ & 3 & 6 & 7 & 8 & 24 \\
$45-54$ & 3 & 9 & 8 & 5 & 25 \\
$\geqslant 55$ & 14 & 36 & 32 & 22 & 104 \\
Total & & & & & \\
Females & 2 & 9 & 2 & 1 & 14 \\
$\leqslant 24$ & 5 & 10 & 4 & 5 & 24 \\
$25-34$ & 2 & 7 & 8 & 6 & 23 \\
$35-44$ & 2 & 7 & 4 & 6 & 19 \\
$45-54$ & 1 & 6 & 5 & 9 & 21 \\
$\geqslant 55$ & 12 & 39 & 23 & 27 & 101 \\
Total & & & & & \\
\hline
\end{tabular}


measured to the nearest $5 \mathrm{~mm}$ using a wall-mounted stadiometer.

Whole-body density. The subject's weight was measured in air and in water, with corrections for the density of water. The volume of air in the lungs at the time of the recording of the underwater weight was measured using a He dilution technique (corrected for temperature and pressure) and the volume of gas in the gastrointestinal tract was assumed to be $100 \mathrm{ml}$. Hence the volume of the body was calculated. The proportion of body fat was calculated using Siri's (1956) equation which assumes that the density of body fat is $0.9 \mathrm{~kg} / \mathrm{l}$ and the density of fat-free mass is $1 \cdot 1 \mathrm{~kg} / \mathrm{l}$.

Total body water. Body water was measured using an isotope dilution procedure. The subjects received an oral dose of deuterium oxide $(0.5 \mathrm{~g} / \mathrm{kg}$ body weight $)$ and collected a saliva sample beforehand and another $4 \mathrm{~h}$ afterwards. The concentration of deuterium in each sample was measured using i.r. spectroscopy (FTIR Genesis; ATI Unicam, Cambridge, Cambs., UK) and the pool size calculated. The measured pool size was reduced by $4 \%$ to account for the exchange of deuterium with non-aqueous $\mathrm{H}$. The hydration fraction of fat-free mass was assumed to be 0.7194 (Siri, 1961) and fat mass was calculated as the difference between fat-free mass and body weight.

Dual-energy X-ray absorptiometry. A whole-body DXA scan was performed using a Hologic QDR-1000W scanner (Hologic Inc., Waltham, MA, USA) and analysed using the enhanced version of the software to estimate bone mineral (subsequently used to derive 'ash'), fat and fat-free soft tissue mass.

Four-compartment model. The data collected in the measurements described earlier were also combined to yield an estimation of body fat mass from a four-compartment model as previously derived (Fuller et al. 1992):

$$
\text { fat }(\mathrm{kg})=2.747 \mathrm{BV}-0.710 \mathrm{TBW}+1.460 \mathrm{~A}-2.050 \mathrm{Wt} \text {, }
$$

where BV is body volume (litres), TBW is total body water (litres), A is ash ( $\mathrm{kg})$ and $\mathrm{Wt}$ is body weight $(\mathrm{kg})$.

Skinfold thicknesses. Skinfold thicknesses were measured using Harpenden calipers at four sites (triceps, biceps, subscapula and suprailiac crest) on the non-dominant side of the body. Predicted body density was calculated using the age- and sex-specific prediction equations of Durnin \& Womersley (1974), based on the sum of the skinfold thicknesses at each site. The proportion of body fat was calculated from body density using the equation of Siri (1956).

Tetrapolar bioelectrical impedance analysis. Conventional tetrapolar bioelectrical impedance analysis was measured using the Bodystat-1500 system (Bodystat, Douglas, Isle of Man, UK) with electrodes placed at the standard sites on the hand and foot. The measurement was made immediately following the DXA scan to ensure that all subjects had been supine for $20 \mathrm{~min}$ to minimize potential errors from acute shifts in fluid distribution. Body fat was calculated according to the algorithm provided by the manufacturers. This particular equation was chosen because in a previous analysis it had been demonstrated to be superior to many other impedance-based prediction equations based on its agreement with reference methods (Fuller et al. 1994).

Tanita body-fat analysis. Bioelectrical impedance from foot to foot was measured using the Tanita-305 body-fat analyser (Tanita Corp., Tokyo, Japan) which provides a print-out of measured impedance and calculated body fat. Subjects stood on the metal sole plates of the machine wearing only their swimwear. All measurements were made after a period of at least $10 \mathrm{~min}$ standing to minimize potential errors from acute shifts in fluid distribution. Body composition for all subjects was estimated using the standard prediction equations rather than those designated for athletes, regardless of the exercise habits of the participants. However, as would be expected for a general population sample, very few subjects would have warranted a specialist classification. Details of the prediction equations were provided by the manufacturer. The prediction equation for men is derived against body density (BD) thus:

$\mathrm{BD}=1 \cdot 100696-0.107903 \times \mathrm{Wt} \times \mathrm{Z} / \mathrm{Ht}^{2}+0.00017 \times \mathrm{Z}$,

where Wt is weight $(\mathrm{kg}), \mathrm{Ht}$ is height $(\mathrm{m})$ and $\mathrm{Z}$ is impedance $(\Omega)$, and percentage fat is calculated fom body density as:

$$
\% \text { fat }=(4 \cdot 57 / \mathrm{BD}-4 \cdot 142) \times 100 .
$$

For women the prediction equation estimates fat-free mass (FFM) as:

$$
\begin{aligned}
\mathrm{FFM}(\mathrm{kg})= & 13.96674+0.348613 \times \mathrm{Ht}^{2} / \mathrm{Z} \\
& +0.168998 \times \mathrm{Wt},
\end{aligned}
$$

and percentage fat is calculated as:

$$
(\mathrm{Wt}-\mathrm{FFM}) / \mathrm{Wt} \times 100 .
$$

Weight and height formulas. The proportion of body fat was also estimated from two prediction equations based on weight and height alone (Black et al. 1983; Garrow \& Webster, 1985) which are shown here. In each case relative body weight is calculated as the BMI (weight $/$ height $^{2}$ ).

BMI-1 (Black et al. 1983):

$$
\begin{aligned}
\text { male } \% \text { fat } & =(1.281 \times \mathrm{BMI})-10.13, \\
\text { female } \% \text { fat } & =(1.480 \times \mathrm{BMI})-7.00
\end{aligned}
$$

BMI-2 (Garrow \& Webster, 1985):

$$
\begin{aligned}
\text { male fat mass }(\mathrm{kg})= & ((0.715 \times \mathrm{BMI}) \\
& -12.1) \text { height }^{2}(\mathrm{~m}), \\
\text { female fat mass }(\mathrm{kg})= & ((0.713 \times \mathrm{BMI}) \\
& -9.74) \operatorname{height}^{2}(\mathrm{~m}) .
\end{aligned}
$$

\section{Data analysis}

The measured percentage body fat and weight of fat $(\mathrm{kg})$ from each individual method were compared with the measurement of fat obtained from the four-compartment model, using correlation analysis across the group as a whole and an assessment of the bias and limits of agreement for individual subjects. Data are given for all subjects and sub-divided by sex. The bias between methods in terms of 

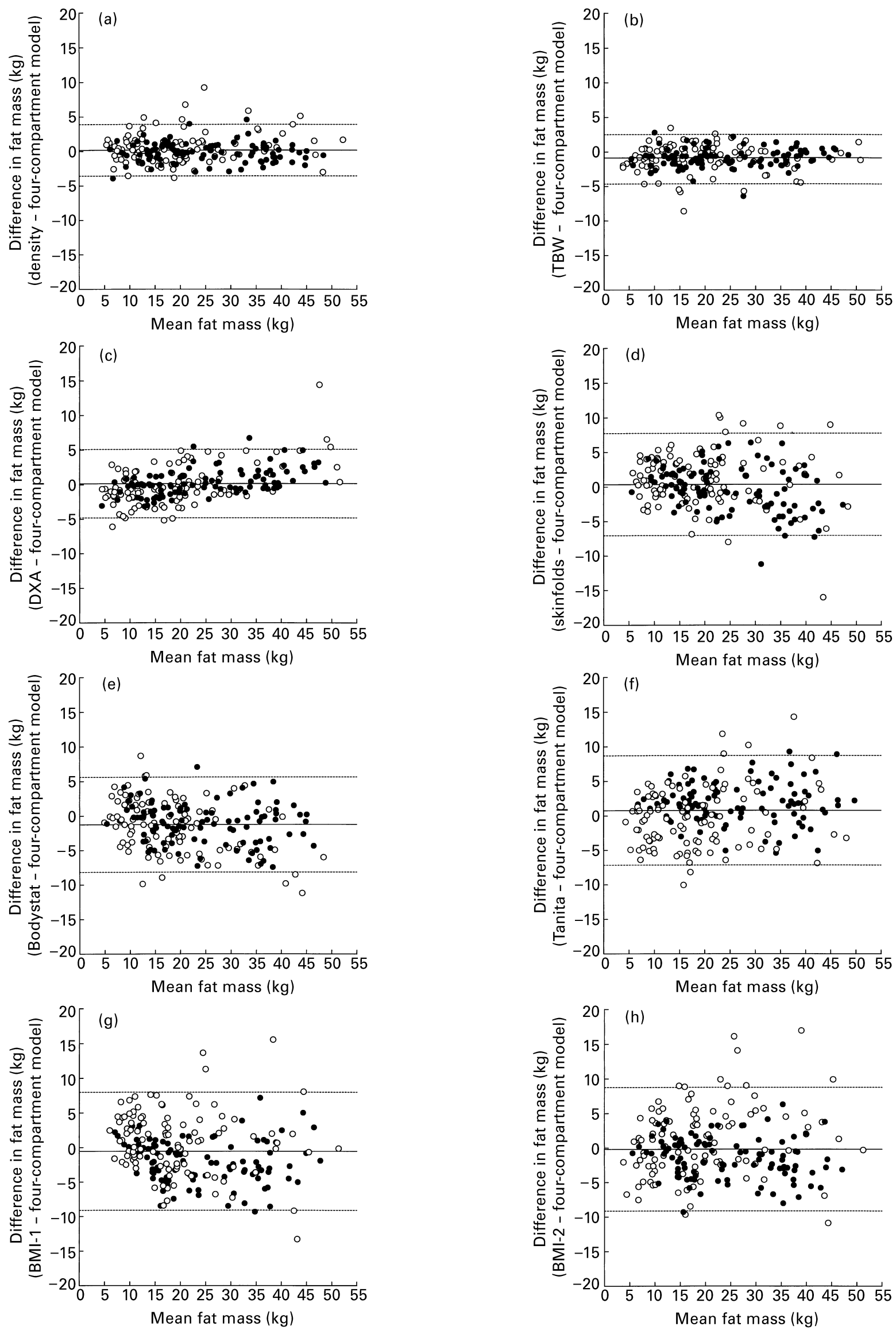
Table 3. Correlation coefficients ( $r$ values) for body fat by all methods relative to a four-compartment model ${ }^{*}$

\begin{tabular}{lcccc}
\hline & & \multicolumn{3}{c}{ Body fat (\%) } \\
\cline { 3 - 5 } Method & $\begin{array}{c}\text { Body fat }(\mathrm{kg}) \\
\text { All }\end{array}$ & All & Male & Female \\
\hline Density & 0.986 & 0.974 & 0.954 & 0.972 \\
TBW & 0.989 & 0.980 & 0.967 & 0.976 \\
DXA & 0.978 & 0.968 & 0.939 & 0.971 \\
Skinfolds & 0.944 & 0.903 & 0.854 & 0.867 \\
Bodystat & 0.952 & 0.901 & 0.818 & 0.881 \\
Tanita & 0.933 & 0.889 & 0.810 & 0.854 \\
BMl-1 & 0.924 & 0.862 & 0.759 & 0.863 \\
BMI-2 & 0.928 & 0.849 & 0.746 & 0.875 \\
\hline
\end{tabular}

TBW, total body water; DXA, dual-energy X-ray absorptiometry.

*For details of methods, see pp. 116-119.

fat-free mass will be equal and opposite to that given for fat mass and the limits of agreement are similar.

In order to develop a best-fit algorithm for the Tanita instrument the subjects were randomly divided into a 'training' set, which included two-thirds of the sample, on which regression models were developed for male and female subjects separately, and a 'test' set, which contained the outstanding third of the sample, on which the performance of the models was tested. The aim of the analysis was to develop a regression model consisting of sex, age, weight, height, body fat estimated by four-compartment analysis, and the impedance measured by the Tanita body-fat analyser. The model takes into account issues such as the normality of the distribution of the dependent variable, the linearity and homoscedasticity of its relationship with each of the independent variables, and the possible presence of interactions between variables.

Preliminary analyses comparing body-fat mass, percentage body fat and body weight on the original and log-transformed scales showed a clear advantage for log body-fat mass adjusted for log body weight. This log transformation is useful in several ways. It removes skewness in variables whose distributions have a long right tail, it can linearize relationships with variables that on the original scale are curved and, if both body fat and weight are logged, the regression model is essentially the same for body fat in $\mathrm{kg}$ and as a percentage of body weight. In addition it allows the fit of ratio indices such as BMI or height ${ }^{2} /$ impedance to be compared with more general indices of the same form (e.g. weight/height ${ }^{\mathrm{k}}$ or height ${ }^{\mathrm{k}} /$ impedance).

\section{Results}

\section{Comparison of methods with a four-compartment model}

The correlation between the four-compartment model and all other methods to measure percentage body fat was high ( $>0.85$; Table 3$)$. However, in order to assess the agreement between methods in greater detail the differences
Table 4. Bias and $95 \%$ limits of agreement (2SD) for percentage body fat in male and female subjects for all methods relative to a fourcompartment model (other method minus four-compartment model)*

\begin{tabular}{|c|c|c|c|c|c|c|}
\hline \multirow[b]{2}{*}{ Method } & \multicolumn{2}{|c|}{ All } & \multicolumn{2}{|c|}{ Male } & \multicolumn{2}{|c|}{ Female } \\
\hline & Bias & $2 \mathrm{SD}$ & Bias & $2 \mathrm{SD}$ & Bias & $2 \mathrm{SD}$ \\
\hline Density & $+0 \cdot 2$ & 4.8 & +0.5 & $5 \cdot 2$ & $-0 \cdot 2$ & $4 \cdot 3$ \\
\hline TBW & $-1 \cdot 2$ & 4.4 & $-1 \cdot 1$ & 4.6 & $-1 \cdot 3$ & 4.2 \\
\hline DXA & -0.1 & $6 \cdot 2$ & -0.5 & 6.9 & +0.2 & 5.4 \\
\hline Skinfolds & +0.5 & 9.2 & $+1 \cdot 3$ & 9.0 & -0.3 & 9.5 \\
\hline Bodystat & -1.5 & 9.3 & -1.5 & 9.8 & -1.4 & 8.7 \\
\hline Tanita & +0.9 & $10 \cdot 2$ & -0.9 & $10 \cdot 9$ & $+2 \cdot 7$ & $8 \cdot 1$ \\
\hline BMI-1 & -0.9 & $10 \cdot 8$ & +0.9 & $11 \cdot 1$ & $-2 \cdot 7$ & $10 \cdot 1$ \\
\hline BMI-2 & -0.7 & 11.3 & +0.7 & $12 \cdot 8$ & $-2 \cdot 1$ & 8.9 \\
\hline
\end{tabular}

TBW, total body water; DXA, dual-energy $X$-ray absorptiometry.

*For details of methods, see pp. 116-119.

between techniques in the measurement of body fat for individual subjects were compared. Fig. 1 shows the agreement between individual methods and a four-compartment model for men and women, with the bias and $95 \%$ CI depicted for the entire group. The bias and $95 \%$ confidence limits for men and women independently for percentage body fat are shown in Table 4 and those for fat mass in Table 5. Results for fat-free mass are equal to those for fat mass, although the bias carried the opposite sign. Overall the agreement for women was greater than for men. On average the values for fat measured by density, DXA, skinfolds and the Tanita analyser were higher than those determined by a four-compartment model, whilst total body water, Bodystat and BMI formulas gave lower values. It is clear from the increasing magnitude of the $95 \%$ confidence limits that the agreement between the four-compartment model and reference methods (density, total body water and DXA) is greater than for prediction techniques (skinfold thicknesses, impedance and weight and height indices). The levels of agreement between the Bodystat or Tanita impedance machines $v$. the four-compartment model were similar, especially in women, although for men the agreement was slightly superior for Bodystat relative to Tanita.

\section{Development of best-fit algorithm for Tanita-305}

Following allocation of subjects to the training or test set, values for the age, height, weight, BMI and percentage body fat of the two populations were not significantly different. In the first model the dependent variable was body fat mass (kg) measured by a four-compartment model, and the independent variables were height, weight, age and Tanita impedance, where fat, height and weight were log transformed. For males in the training set (n 70), the percentage of variance $\left(R^{2}\right)$ accounted for by this model was $84.2 \%$. Weight was by far the most important variable compared with the other three, although the regression coefficients of all four variables were highly significantly different from

Fig. 1. Agreement in the measurement of body fat mass between a four-compartment model and (a) body density, (b) total body water (TBW), (c) dual-energy X-ray absorptiometry (DXA), (d) skinfold thickness measurements, (e) Bodystat, (f) Tanita, (g) BMl equation of Black et al. (1983) (BMI-1), (h) BMI equation of Garrow \& Webster (1985) (BMI-2). (- - , Mean bias; (---), $95 \%$ limits of agreement; (O), men; $(\bullet)$, women. 
Table 5. Bias and $95 \%$ limits of agreement (2SD) for fat mass $(\mathrm{kg})$ in male and female subjects for all methods relative to a fourcompartment model (other method minus four-compartment model)*

\begin{tabular}{|c|c|c|c|c|c|c|}
\hline \multirow[b]{2}{*}{ Method } & \multicolumn{2}{|c|}{ All } & \multicolumn{2}{|c|}{ Male } & \multicolumn{2}{|c|}{ Female } \\
\hline & Bias & $2 S D$ & Bias & $2 S D$ & Bias & $2 \mathrm{SD}$ \\
\hline Density & +0.2 & $3 \cdot 7$ & +0.5 & 4.4 & -0.2 & $2 \cdot 8$ \\
\hline TBW & -0.9 & 3.4 & -0.9 & 3.9 & -0.9 & $2 \cdot 7$ \\
\hline DXA & +0.1 & $5 \cdot 0$ & $-0 \cdot 1$ & 5.9 & +0.3 & $3 \cdot 8$ \\
\hline Skinfolds & +0.3 & 7.4 & $+1 \cdot 1$ & 7.9 & -0.4 & $6 \cdot 6$ \\
\hline Bodystat & $-1 \cdot 3$ & 6.9 & -1.4 & $7 \cdot 7$ & $-1 \cdot 1$ & $6 \cdot 0$ \\
\hline Tanita & +0.8 & 7.9 & -0.3 & 9.0 & +1.9 & 5.9 \\
\hline BMl-1 & -0.6 & 8.5 & +0.8 & 9.5 & -1.9 & $6 \cdot 4$ \\
\hline BMI-2 & -0.2 & $9 \cdot 0$ & $+1 \cdot 1$ & 10.5 & -1.5 & $6 \cdot 0$ \\
\hline
\end{tabular}

TBW, total body water; DXA, dual-energy X-ray absorptiometry.

${ }^{*}$ For details of methods, see pp. 116--119.

zero. The residual standard deviation (RSD) was $4.4 \%$. The regression coefficient for ln height was almost exactly twice that for ln weight, indicating that weight and height can be combined as weight $/$ height $^{2}$, i.e. the BMI.

A similar model for the training set of females ( $n$ 66) showed broadly similar results. The main difference was a smaller RSD of $3.9 \%$ which reflected the generally better fit of these data relative to those for males, as previously observed. This resulted in an $R^{2}$ of $80.9 \%$. In addition, for females age was not significant and the ln height coefficient was two-thirds that for the males. The contribution made by the measurement of impedance was also smaller, but equally significant, while ln weight had almost the same coefficient as for males but a much higher significance. This means that the combination of weight and height into a single index corresponds to weight/height ${ }^{1.4}$, showing that the weight-height relationship was weaker in females than males.

Combining the models for the two sexes into a single model and adding interaction terms to test for equality of coefficients between the sexes, showed that height was the only variable which was significantly different. Moreover the ln height and ln weight coefficients were again in the approximate ratio $2: 1$ indicating that they can be replaced by $\ln$ BMI.

The performance of the regressions was tested by applying them to the subjects in the test set. The agreement of the new regressions with a four-compartment model is not directly comparable with the agreement observed between other methods since the equation has been developed and tested in the same group. However the mean agreement for men was 0.04 (2SD 0.50) kg fat and for women 0.04 (2SD $0 \cdot 26) \mathrm{kg}$ fat. In each case the predicted percentage body fat was compared with the observed value and the mean and standard deviation of the residual were obtained (observed-predicted). The mean residual bias was $+0.3 \%$ for males and $+1.5 \%$ for females and the standard deviations were $4.8 \%$ for males and $3.3 \%$ for females. The standard deviations were similar to the RSD values obtained with the training set. This justifies expanding the regression model to the whole data set, training and test sets combined, for both sexes. When this was done all the variables were highly significant, adjusted $R^{2}$ was $84.9 \%$, and the RSD was $4.1 \%$. Thus the final model which is applicable to both sexes is:

$$
\begin{aligned}
\% \text { body fat }= & -156 \cdot 1-89 \cdot 1 \ln (\text { height })+45 \cdot 6 \ln (\text { weight }) \\
& +0 \cdot 120 \text { age }+0.0494 \mathrm{Z}+(19 \cdot 6 \ln (\text { height }) \\
& (\text { for females })) .
\end{aligned}
$$

where height is in $\mathrm{cm}$, weight in $\mathrm{kg}$ and age in years. The weight-height relationship is very close to the BMI and for simplicity the equation may be approximated to:

$$
\begin{aligned}
\% \text { body fat }= & -573.8+45.5 \ln (\mathrm{BMI}) \\
& +0.118 \text { age }+0.0492 \mathrm{Z} \\
& +(19.3 \ln (\text { height })(\text { for females })) .
\end{aligned}
$$

The addition of the impedance measurement to the final model increased the adjusted $R^{2}$ from $79.3 \%$ to $85 \%$.

\section{Comparison of Tanita and a conventional impedance system (Bodystat)}

In order to compare the Tanita system with a conventional impedance system (Bodystat) a similar exercise was conducted using the Bodystat data to derive a 'best-fit' regression. Here the mean residual bias in the test data set was $-0 \cdot 1 \%$ for males and $+1.2 \%$ for females and the standard deviations were $4.3 \%$ for males and $3.1 \%$ for females. This demonstrates a very minor improvement relative to the Tanita system.

\section{Discussion}

This study provides a comprehensive evaluation of a range of in vivo body composition methods against a reference four-compartment model in a large and heterogeneous population sample and extends the previous studies by Nunez et al. (1997), Bell et al. (1998) and Utter et al. (1999). The study of Nunez et al. (1997) used a comparable population and compared the data against individual measurements of body composition by DXA, underwater weighing and tritium dilution. They demonstrate a similar level of agreement for the novel Tanita system as conventional bioimpedance. Bell et al. (1998) studied a group of only fifty-seven subjects and compared the body water measured by deuterium dilution with that predicted from the Tanita body fat analyser. At a group level there was good agreement, but there was evidence of an increasing underestimation of body water by impedance as the body water volume increased. Utter et al. (1999) assessed the accuracy of the Tanita system in women in a cross-sectional study and in a subgroup of obese women before and after weight loss. They found no significant difference between underwater weighing and the Tanita body fat analyser in either the cross-sectional or longitudinal study. However in each case the conclusions of the study are limited by the use of a twocompartment model (or in the case of DXA, a threecompartment model) rather than the more sophisticated four-compartment approach.

Moreover, in the present study we examined the accuracy of the Tanita system in the context of a range of other body composition measurements to provide a comprehensive 
dataset to evaluate the different methods. The accuracy of the reference methods (density, total body water and DXA) was clearly superior to that of any of the prediction techniques. Among the prediction techniques, skinfold thicknesses and the impedance systems were very similar and superior to either of the BMI formulas. The limits of agreement for fat mass for the Tanita body fat analyser $( \pm 7.9 \mathrm{~kg})$ were slightly greater than for the the Bodystat impedance system $( \pm 6.9 \mathrm{~kg}$ ), although the bias was smaller (Tanita $0.8 \mathrm{~kg}$, Bodystat $-1.3 \mathrm{~kg}$ ). Thus, using the existing regression equations there is little to recommend one impedance system over and above the other. The variability observed in the data was due in part to measurement error and in part to biological differences between subjects in the extent to which their body composition deviated from the assumptions of the methods. It should be noted that differences in the assumptions made in the calculation of body fat from reference techniques and differences in the prediction equations will yield slightly different values for the bias observed relative to a four-compartment model, but the limits of agreement will be less affected.

We have used this dataset to develop a novel prediction equation to estimate fat mass from the Tanita body-fat analyser. In doing so we have returned to basic mathematical principles rather than following the traditional format for impedance-based prediction equations (Hoffer et al. 1969). Our approach incorporates two novel elements which warrant consideration. First, we have used a logarithmic transformation to make allowances for the distribution of the dependent variable. Second, each variable has been entered into the model independently to select the best-fit; specifically height and impedance have been entered as independent terms, rather than constraining the model to the term height ${ }^{2} /$ impedance. Although height ${ }^{2}$ has traditionally been used as a proxy for conductor length this assumption can legitimately be challenged, particularly in relation to the foot-to-foot measurement of impedance made by the Tanita body-fat analyser.

We have extended this analysis to generate a novel bestfit algorithm for the conventional tetrapolar impedance system (represented by the Bodystat machine) to evaluate the relative performance of the two methods. Under these conditions the performance of the two impedance methods was very similar, suggesting that the enhanced simplicity of the novel Tanita method is not associated with a clinically significant decrement in performance relative to the conventional system. This may be explained by two factors; first in both systems body weight dominates the prediction of body fat while the impedance measurement makes only a modest additional contribution and second, in the conventional tetrapolar system, the contribution of body segments to the measured impedance is disproportionate to their mass, with the legs contributing $44 \%$ of the measured impedance (Fuller \& Elia, 1989).

In summary, the Tanita body fat analyser is a valid alternative method to other impedance-based prediction techniques for the measurement of body fat. The minor differences in accuracy between the two approaches to the measurement of body composition by impedance suggests that there is no clear advantage to using the traditional tetrapolar surface electrodes on the hand and foot over and above the novel stand-on system. The latter has clear practical advantages in many situations, not least for people who wish to measure their body fat at home on a regular basis. However, in common with other simple field methods the accuracy of the measurement in individuals, relative to a four-compartment model, is poor. Further studies are underway to determine the absolute accuracy of the method for the measurement of changes in body fat, since this may be the variable of particular interest in some nutritional studies or for individuals using measurements of body composition at home to assess their progress in a weight management programme.

\section{Acknowledgement}

This study was sponsored by Tanita Ltd.

\section{References}

Bell NA, McClure PD, Hill RJ \& Davies PSW (1998) Assessment of foot-to-foot bioelectrical impedance analysis for the prediction of total body water. European Journal of Clinical Nutrition 52, 856-859.

Black D, James WPT, Besser GM, Brook CGD, Craddock D, Garrow JS, Hockaday TDR, Lewis B, Pilkington TRE, Silverstone JT, Mann JI, Miller DD, Pyke DA, Williams DG \& Skinner RK (1983) Obesity. A report of the Royal College of Physicians. Journal of the Royal College of Physicians 17, 5-65.

Durnin JVGA \& Womersley J (1974) Body fat assessed from total body density and its estimation from skinfold thickness measurement in 481 men and women aged 16 to 72 years. British Journal of Nutrition 32, 77-97.

Fuller NJ \& Elia M (1989) Potential use of the bio-electrical impedance of the 'whole-body' and of body segments for the assessment of body composition: comparison with densitometry and anthropometry. European Journal of Clinical Nutrition 43, 779-791.

Fuller NJ, Jebb SA, Laskey MA, Coward WA \& Elia M (1992) Four component model for the assessment of body composition in humans: comparison with alternative methods and evaluation of the density and hydration of fat free mass. Clinical Science 82, 687-693.

Fuller NJ, Sawyer MB \& Elia M (1994) Comparative evaluation of body composition methods and predictions, and calculation of density and hydration fraction of fat-free mass, in obese women. International Journal of Obesity 18, 503-512.

Garrow JS \& Webster J (1985) Quetelet's Index as a measure of fatness. International Journal of Obesity 9, 147-153.

Hoffer EC, Meador CK \& Simpson DC (1969) Correlation of whole-body impedance with total body water volume. Journal of Applied Physiology 27, 531-534.

Jebb SA (1997) Measurement of soft tissue composition by dual energy X-ray absorptiometry. British Journal of Nutrition 77, $151-163$.

Jebb SA (1998) Measuring body composition: from the laboratory to the clinic. In Clinical Obesity, pp. 18-49 [PG Kopelman and MJ Stock, editors]. London: Blackwell.

Jebb SA \& Elia M (1993) Techniques for the measurement of body composition: a practical guide. International Journal of Obesity 17, 611-621.

Jebb SA \& Elia M (1995) Multicompartment models in health and disease. In Body Composition Techniques in Health and Disease, pp. 240-254 [PSW Davies and TJ Cole, editors]. Cambridge: Cambridge University Press. 
Jebb SA, Murgatroyd PR, Goldberg GR, Prentice AM \& Coward WA (1993) In vivo measurement of changes in body composition: description of methods and their validation against 12-d continuous whole-body calorimetry. American Journal of Clinical Nutrition 58, 455-462.

Nunez C, Gallagher D, Visser M, Pi-Sunyer FX, Wang Z \& Heymsfield SB (1997) Bioimpedance analysis: evaluation of leg-to-leg system based on pressure contact foot-pad electrodes. Medicine and Science in Sports and Exercise 29, 524-531.
Siri WS (1956) The Gross Composition of the Body. New York, NY: Academic Press.

Siri WS (1961) Body Composition From Fluid Spaces and Density: a Combined Analysis of Methods. Washington, DC: National Academy of Sciences - National Research Council.

Utter AC, Nieman DC, Ward AN \& Butterworth DE (1999) Use of the leg-to-leg bioelectrical impedance method in assessing body composition change in obese women. American Journal of Clinical Nutrition 69, 603-607. 